\title{
Positive Affect and Flexibility: Overcoming the Precedence of Global over Local Processing of Visual Information
}

\author{
Nicola Baumann ${ }^{1,2}$ and Julius Kuhl ${ }^{1}$
}

\begin{abstract}
Previous findings on the relationship between positive mood and global processing are often based on visual matching tasks that involve a choice between global and local strategies. Preferences for global processing in positive mood, however, do not imply a reduced ability to process locally. The present experiment tested the assumption that positive affect increases flexibility in cognitive processing as indicated by the ability to overcome global precedence, and to respond rapidly to non-dominant (local) features when the task necessitates it. Consistent with expectations, participants responded significantly faster to local targets after positive compared to neutral and negative prime words. The typical precedence of global over local processing observed after neutral and negative prime words was reversed after positive prime words. Findings support the assumption that positive affect increases cognitive flexibility. Furthermore, findings suggest that mood-related preferences in global versus local processing cannot be generalized to processing ability.
\end{abstract}

KEY WORDS: mood; positive affect; negative affect; local processing; global processing; PSI theory.

It is widely accepted in the psychological literature that there is a relationship between emotions and the way individuals think and process information. However, the nature of this relationship is controversial. One line of research suggests that emotions are used as information about the value of accessible information and influence the "level of focus" (Clore, Gasper, \& Garvin, 2001). According to this view, positive compared to negative affect promotes greater reliance on accessible information such as stereotypes (Bodenhausen, Kramer, \& Susser, 1994), heuristic rather than systematic processing strategies (Schwarz, 2002), and global

\footnotetext{
${ }^{1}$ University of Osnabrück, Germany.

${ }^{2}$ Address all correspondence to Nicola Baumann, Department of Psychology, University of Osnabrück, Seminarstr. 20, 49069 Osnabrück, Germany; e-mail: nbaumann@uos.de.
} 
rather than local features of visual information (Gasper \& Clore, 2002). Another line of research suggests that positive affect leads to greater flexibility in cognitive processing (Dreisbach \& Goschke, 2004; Isen, 2004) as evidenced in more creative problem solving of the Duncker candle problem (cf. Isen, 1999b), less anchoring and more integration of information in medical decision-making (Estrada, Isen, \& Young, 1997), greater ability to switch perspectives and find viable solutions in negotiation (cf. Isen, 2001), and an increased ability to enact intentions against strong habitual tendencies (Kuhl \& Kazén, 1999). According to this "flexibility" hypothesis, positive affect does not necessarily promote accessible or global processing but facilitates open, flexible and efficient processing.

In the present article we examine the flexibility hypothesis, which suggests that positive affect does not necessarily promote a focus on global features (the forest) but can facilitate the processing of local features (the trees). Because processing of global features takes precedence over local features (Navon, 1977, 1983; for a review see Kimchi, 1992) attending to the tree requires flexibility to overcome the dominant, more accessible strategy of attending to the forest. We assume that positive affect promotes this flexibility to shift to non-dominant, local features of visual stimuli if the task requires such a shift. To our knowledge, this hypothesis has not been tested so far.

The flexibility hypothesis can be derived from Personality Systems Interaction (PSI) theory (Kuhl, 2000, 2001). According to this theory, moderate levels of positive affect increase the activation of a central executive system called extension memory whereas unattenuated negative affect reduces access to this system. Extension memory is conceived of as an implicit representational system that is necessary to have an overview of extended semantic fields (Rotenberg, 1993), relevant episodes experienced (Wheeler, Stuss, \& Tulving, 1997) and integrated self-representations (Koole \& Kuhl, 2003; Kuhl, 2000). It operates according to connectionist principles and promotes intuitive-holistic processes (Baumann \& Kuhl, 2002; Bolte, Goschke, \& Kuhl, 2003; Beeman et al., 1994). According to PSI theory, extension memory is a high-level intuitive system that can be distinguished from a low-level intuitive system (cf. Epstein, Pacini, Denes-Raj, \& Heier, 1996) which is involved in intuitive (i.e., automatized) behavior control, according to PSI theory. Whereas the low-level intuitive system may promote greater reliance on accessible information and dominant reactions, the high-level intuitive system (extension memory) promotes access to extended networks of relevant semantic meanings, remote action alternatives and integrative abilities (Baumann \& Kuhl, 2002; Koole \& Jostmann, 2004). For example, in positive-affect conditions participants give more unusual and diverse word associations (Isen, Johnson, Mertz, $\&$ Robinson, 1985), detect coherence among weakly associated words (Bolte et al., 2003), and seek variety among safe, enjoyable products (Kahn \& Isen, 1993). These effects of positive affect can be interpreted as examples of the workings of extended semantic networks. Furthermore, they are consistent with the assumption that positive affect facilitates access to extension memory. 
According to PSI theory, extension memory provides a great number of internal states such as needs, preferences, feelings and action alternatives that can be taken into account simultaneously for decision-making. This extended scope enables the person to choose goals that satisfy multiple constraints and to feel priorities and make decisions among a variety of options without having to scrutinize each of them in a step-by-step, conscious manner. The intuitiveholistic processing characteristic of extension memory may be associated with a focus on global processing in many situations. However, because extension memory provides action alternatives, activation of extension memory is expected to increase the flexibility to overcome the precedence of the dominant response mode (e.g., global processing) if the task requires an alternative mode (i.e., local processing). Moderate levels of positive affect seem especially apt to stimulate cognitive flexibility (Isen, 1999a) presumably associated with extension memory.

At first glance, previous findings revealing a positive relationship between positive mood and global processing seem to contradict our hypothesis (Basso, Schefft, Ris, \& Dember, 1996; Gasper \& Clore, 2002). However, observed preferences for global processing when given a free choice between global and local strategies do not necessarily imply an impaired ability to switch to local processing when the task requires such a shift. For example, Gasper and Clore (2002) used a visual matching test (Kimchi and Palmer, 1982) in which participants have to indicate which one of two sample figures looks most like a target figure. Each figure was either a square or a triangle (global feature) made up of smaller squares or triangles (local feature). As predicted, participants in whom positive mood was induced or naturally present (i.e., within the neutral condition) were more likely to match the ambiguous objects on the basis of global features than participants in a negative-mood condition. However, the finding that participants use the easily accessible, global feature in the matching task when being in a positive mood does not imply an impaired ability to focus on the less accessible, local feature. Notice that the task did not suggest using the less accessible route. A similar argument can be made regarding findings (Basso et al., 1996) that demonstrate a relationship between trait happiness or optimism and a global bias. We conducted the following experiment to test whether preferences for global processing observed in a positive mood condition imply an impaired ability to use local processing.

\section{EXPERIMENT}

In order to test the hypothesis that positive affect promotes flexibility to shift the focus to non-dominant (local) stimulus features, we assessed reaction times in a target detection paradigm. Our global-local shape task consisted of single figures that unambiguously contained a given target shape (see Fig. 1) or not. We used an affective priming procedure in order to test mood effects within participants. Our flexibility hypothesis predicts reduced reaction times for local targets after positive compared to neutral and negative prime words. In contrast, negative compared to 

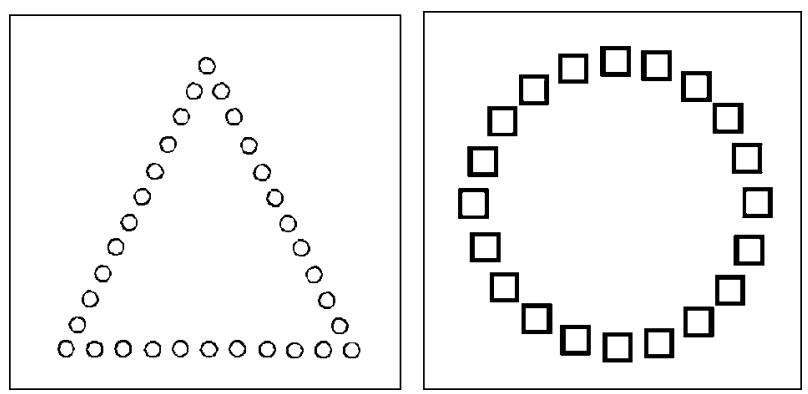

Fig. 1. Two sample items from the global-local shape task. In the left item, the target shape "circle" is present on a local dimension. In the right item, the target shape is present on a global dimension. Other items did not contain the target shape (e.g., a square composed of smaller triangles).

neutral prime words are expected to increase reaction times for local targets. The typical precedence of global over local targets is expected to occur after neutral and negative prime words but not after positive prime words.

Positive affect is expected to reduce reaction times for local targets because it increases access to the system that provides action alternatives and thus facilitates a shift to the non-dominant response alternative. Although global processing is pressumed to be the default focus for most participants (Navon, 1977, 1983), individuals may differ in their default focus due to differences in viewing distance or individual preferences for global versus local processing. Therefore, individuals who show a precedence of local over global processing after neutral primes are expected to show facilitated target detection after positive primes when targets are presented on the non-dominant global dimension. To summarize, positive affect is not expected to support a specific processing focus but to increase the flexibility to shift to the non-dominant response alternative. Whether local or global aspects are the non-dominant response alternatives may vary between individuals.

\section{METHOD}

\section{Participants}

Seventy-eight participants ( 40 women and 38 men) were recruited through flyers at the university of Osnabrück. Their mean age was 24 years (range 15-57). Participants received $10 €$ (about $\$ 12$ ) in return for their participation.

\section{Materials and Procedure}

At the beginning of each session participants were asked to generate affective prime words. They were to recall personal life events that had made them feel either 
"happy and positive" or "sad and negative." They were to generate six positive and six negative words that would remind them of the original experiences and elicit the associated feelings. Finally, participants marked six neutral words from a given list of 30 words that had received neutral ratings in a pilot study. The experimenter typed in individual prime words while participants worked on a filler questionnaire. Previous studies demonstrated the functional equivalence of self-generated and standard prime words as well as non-verbal, figurative primes (Kazén \& Kuhl, in press; Kuhl \& Kazén, 1999). Whereas positive and negative prime words may differ according to features unrelated to their affective content (e.g., word length, language frequency, concreteness, associative value, etc.), these alternative explanations of stimulus effects can be ruled out with non-verbal primes. The finding that self-generated prime words were functionally equivalent to non-verbal primes supports the assumption that positive and negative self-generated prime words are able to produce effects that are attributable to their affective content.

Similar to the material used by Derryberry and Reed (1998), the shape task consisted of geometrical objects (circle, triangle, square, or diamond) composed of smaller geometrical objects (see Fig. 1). Participants' task was to decide whether a given target shape was present or not. For example, the target shape "circle" is present on the local dimension in the left sample item of Fig. 1 whereas it is present on the global dimension in the right sample item. In either cases, the correct answer is "yes." Each trial of the shape-detection task consisted of an individual prime word (400 ms), an inter-stimulus-interval $(500 \mathrm{~ms})$, and a target item. There was a variable inter-trial-interval (500-3000 ms). Participants were asked to respond as rapidly and accurately as possible to target items. Personal prime words were introduced as a means to facilitate attention for subsequent tasks. Participants were asked to attend to these words and recall associated feelings. In order to further increase attention to prime words, at random intervals participants were asked to classify the affective valence of the prime. The shape-detection task consisted of a total of 156 trials (including 12 practice trials): $50 \%$ did not include the target shape, $25 \%$ included it on the global dimension, and $25 \%$ on the local dimension. Four target shapes (circle, triangle, square, or diamond) were alternated block-wise. Within blocks, the presence/absence and global/local dimension of target shapes as well as the affective valence of prime words were completely balanced. Positive negative, and neutral prime words were presented in random order. Experimental sessions lasted about $60 \mathrm{~min}$.

\section{RESULTS}

\section{Response Latencies}

Response latencies for correct target detections were analyzed using a 2 (Target Dimension: local vs. global) $\times 3$ (Prime Type: positive vs. neutral vs. 
Table I. Mean RT (ms) for Correct Responses and Mean Error Rate (\%) as a Function of Target Dimension and Prime Type

\begin{tabular}{|c|c|c|c|c|c|c|}
\hline & \multicolumn{3}{|c|}{ Latencies } & \multicolumn{3}{|c|}{ Error rates } \\
\hline & Neutral & Positive & Negative & Neutral & Positive & Negative \\
\hline \multicolumn{7}{|l|}{ Global } \\
\hline$M$ & $958_{a}$ & $999_{a}$ & $981_{a}$ & $5.13_{a}$ & $8.01_{b}$ & $5.02_{a}$ \\
\hline$S D$ & 277 & 275 & 280 & 7.51 & 9.25 & 7.75 \\
\hline \multicolumn{7}{|l|}{ Local } \\
\hline$M$ & $996_{a}$ & $940_{b}$ & $1098_{c}$ & $4.70_{a}$ & $5.66_{a, b}$ & $7.16_{b}$ \\
\hline$S D$ & 274 & 265 & 307 & 7.67 & 6.78 & 8.78 \\
\hline
\end{tabular}

Note. Means in the same row that do not share subscripts differ at $p<.05$ using a Bonferroni correction. Means in the same column printed in italic differ at $p<.05$ using a Bonferroni correction.

negative) analysis of variance design (ANOVA), with repeated measures on both factors. The ANOVA yielded a significant main effect of Target Dimension, $F(1,77)=6.76, p<.02$. Consistent with the global-precedence assumption, participants responded significantly faster to global than local targets (980 vs. $1010 \mathrm{~ms})$. In addition, there was a significant main effect of Prime Type, $F(2,154)=22.22, p<.001$. Response latencies were significantly increased after negative (1039 ms) compared to neutral $(977 \mathrm{~ms})$ and positive primes $(970 \mathrm{~ms})$. More importantly, there was a highly significant Target Dimension $\times$ Prime Type interaction, $F(2,154)=28.55, p<.001$ (see Table I, left columns). Consistent with expectations, response latencies to local targets were significantly reduced after positive compared to neutral and negative primes. That is, positive prime words facilitated a shift to the non-dominant (local) response alternative. In contrast, response latencies to local targets were significantly increased after negative compared to neutral primes. Dependent t-tests confirm the typical precedence of global over local processing after neutral and negative primes: Participants responded significantly more slowly to local, compared to global, targets after neutral primes, $t(77)=2.16, p<.05$, and after negative primes, $t(77)=5.86$, $p<.001$. Somewhat unexpectedly, the precedence of global over local processing was not only absent after positive primes but even significantly reversed: Participants responded significantly faster to local, compared to global, targets after positive primes, $t(77)=-3.41, p<.002$.

\section{Individual Differences in Default Focus}

Because persons can differ in their default focus we used reaction times after neutral primes to estimate participants' default focus. After neutral primes, 52 participants had significantly shorter reaction times for global compared to local targets (see upper half of Table II). In this "global default group," response 
Table II. Mean RT (ms) for Correct Responses and Mean Error Rate (\%) as a Function of Default Focus, Target Dimension and Prime Type

\begin{tabular}{|c|c|c|c|c|c|c|}
\hline & \multicolumn{3}{|c|}{ Latencies } & \multicolumn{3}{|c|}{ Error rates } \\
\hline & Neutral & Positive & Negative & Neutral & Positive & Negative \\
\hline \multicolumn{7}{|c|}{ Global default group $(N=52)$} \\
\hline \multicolumn{7}{|c|}{ Dominant (global) } \\
\hline$M$ & $913_{a}$ & $1010_{b}$ & $944_{a}$ & $4.49_{a}$ & $7.53_{b}$ & $4.65_{a}$ \\
\hline$S D$ & 267 & 305 & 256 & 5.59 & 8.46 & 6.69 \\
\hline \multicolumn{7}{|c|}{ Non-dominant (local) } \\
\hline M & $1033_{a}$ & $951_{b}$ & $1098_{c}$ & $4.49_{a}$ & $5.77_{a, b}$ & $7.21_{b}$ \\
\hline$S D$ & 286 & 262 & 310 & 7.46 & 6.71 & 8.58 \\
\hline \multicolumn{7}{|c|}{ Local default group $(N=26)$} \\
\hline \multicolumn{7}{|c|}{ Dominant (local) } \\
\hline$M$ & $921_{a}$ & $917_{a}$ & $1099_{b}$ & $5.13_{a}$ & $5.45_{a}$ & $7.05_{a}$ \\
\hline$S D$ & 237 & 274 & 309 & 8.19 & 7.05 & 9.34 \\
\hline \multicolumn{7}{|c|}{ Non-dominant (global) } \\
\hline M & $1049_{a}$ & $977_{b}$ & $1054_{a}$ & $6.41_{a}$ & $8.97_{a}$ & $5.77_{a}$ \\
\hline$S D$ & 278 & 206 & 316 & 10.36 & 10.78 & 9.65 \\
\hline
\end{tabular}

Note. Means in the same row that do not share subscripts differ at $p<.05$ in paired comparisons using a Bonferroni correction. Means in the same column printed in italic differ at $p<.05$ using a Bonferroni correction.

latencies to non-dominant local targets were significantly reduced after positive primes compared to neutral and negative primes. In addition, response latencies to non-dominant local targets were significantly increased after negative, compared to neutral, primes. The Target Dimension $\times$ Prime Type interaction was significant, $F(2,100)=35.81, p<.001$. In contrast, 26 participants had significantly shorter reaction times for local compared to global targets (see lower half of Table II). In this "local default group," response latencies to non-dominant global targets were significantly reduced after positive primes compared to neutral and negative primes. The difference between neutral and negative primes was not significant. The Target Dimension $\times$ Prime Type interaction was significant, $F(2,52)=10.73, p<.001$. To summarize, in both default groups, positive affect facilitated shifts to the non-dominant response alternatives.

\section{Error Rates}

To rule out an alternative interpretation of response latencies in terms of a speed-accuracy trade-off, error rates were analyzed. The 2 (Target Dimension) $\times 3$ (Prime Type) ANOVA yielded a significant main effect of Prime Type, $F(2,154)=$ $4.73, p<.02$. Error rates were higher after positive $(6.84 \%)$ and negative $(6.09$ $\%)$ compared to neutral $(4.92 \%)$ primes. More importantly, there was a significant Target Dimension $\times$ Prime Type interaction, $F(2,154)=5.72, p<.005$ (see Table I, right columns). Error rates for global targets were significantly increased 
after positive compared to neutral and negative primes. However, error rates for local targets did not significantly differ after positive compared to neutral and negative primes. Findings do not support a speed-accuracy trade-off interpretation of the short response latencies to local targets observed after positive primes in the total sample.

\section{Individual Differences in Default Focus}

In the "global default group," error rates for non-dominant local targets were not significantly increased after positive compared to neutral and negative primes. In the "local default group," error rates for non-dominant global targets were not significantly increased after positive compared to neutral and negative primes. Findings do not support a speed-accuracy trade-off interpretation of short response latencies to non-dominant target dimensions observed after positive primes in both default groups.

\section{DISCUSSION}

The present experiment tested the assumption that positive affect is associated with increased flexibility in cognitive processing as indicated by the ability to overcome the precedence of global in favor of local processing when the task requires local processing. In order to test this assumption it was necessary to use a target detection paradigm that does not give participants a choice between global and local processing strategies as provided in visual matching tasks (Kimchi \& Palmer, 1982). Matching tasks are likely to assess processing preferences and do not allow conclusions to be drawn concerning cognitive ability. In contrast to a matching task, the shape-detection task used in our experiment requires a shift to the nonpreferred dimension whenever the target shape is represented locally rather than globally. Consistent with expectations, latencies for correct responses to local targets were significantly reduced after positive compared to neutral and negative prime words. An alternative interpretation in terms of a speed-accuracy trade-off was ruled out by convergent findings in error rates. Findings are consistent with the assumption that even short-lived inductions of positive affect are able to foster cognitive flexibility as indicated by the ability to switch to a non-dominant (e.g., local) response alternative. Our data suggest that findings on mood-related processing preferences (e.g., Gasper \& Clore, 2002) cannot be generalized to allow conclusions about corresponding processing abilities.

In addition to the flexibility-enhancing effect of positive affect, the present data also show a flexibility-reducing effect of negative affect: Negative prime words significantly increased latencies to local targets while not slowing latencies 
to global targets. This finding is consistent with PSI theory. According to the negative affect modulation assumption of PSI theory, negative affect reduces access to extension memory and, as a result, reduces cognitive flexibility. Thus, shifting from the default global to the required local features becomes more difficult under conditions of negative affect. The finding suggests that negative affect does not slow down cognitive processing per se, but reduces cognitive flexibility presumably associated with extension memory.

The assumption that affect does not necessarily support a specific processing focus but modulates the ability to shift to non-dominant response alternatives was further supported by the analyses of individual differences. Although there was a precedence of global over local processing for most participants, one third of the sample showed a processing advantage for local targets after neutral primes. In this local default group, positive affect increased the flexibility to shift to the non-dominant global response alternative. In the global default group, in contrast, positive affect increased, whereas negative affect decreased, the flexibility to shift to the non-dominant local response alternative. Findings are consistent with PSI theory, that moderate levels of positive affect activate a system that provides response alternatives (i.e., extension memory) whereas negative affect inhibits extension memory.

Recently, Gasper (2004) tested the idea that mood effects do not occur unless stimuli are ambiguous and the appropriate processing strategies are open to interpretation. From the absence of mood-related global-local differences in reaction times for unambiguous stimuli, Gasper (2004) concluded that ambiguity may be needed for mood effects. The visual matching task used in her study required participants to compare two sample figures with a target figure. Strategies used for those multiple comparisons may vary along other dimensions than global-local. In the present experiment, we obtained highly significant mood-related differences in global versus local processing using unambiguous stimuli. Our data suggest that ambiguity is not a necessary prerequisite for mood effects.

\section{LIMITATIONS AND FUTURE PERSPECTIVES}

Despite the stimulating results, the present research is still preliminary in many ways. First, results were obtained with only one affect induction procedure (i.e., affective priming) and may be restricted to rather short-lived changes in affect. Future research should explore whether the same effects occur for longlasting mood induction procedures. Second, the present experiment used selfgenerated prime words. Positive and negative primes might not be comparable in ways other than their affective tone. Although previous research has demonstrated the functional equivalence of self-generated and standard prime words as well as non-verbal, figurative primes (Kazén \& Kuhl, in press; Kuhl \& Kazén, 1999), 
it would be interesting to replicate the present findings with prime material that allows more experimental control.

Third, the present study used only one stimulus onset asynchrony (SOA) between prime and target $(900 \mathrm{~ms})$. However, the SOA may play an important role in the effects of positive affect (cf. Kazén \& Kuhl, 2005). Therefore, it would be vital to conduct further research on the activation dynamics of positive affect and extension memory.

Finally, the present data are inconsistent concerning the effect of positive prime words on the dominant response alternative. For example, positive affect increased the error rate for the dominant global response alternative (see Table I). In the local default group, however, positive affect did not increase the error rate for the dominant local response alternative (see Table II). Also, positive affect increased latency of correct responding for the dominant response in one instance (the global default group, see Table II) but did not do so in the other two (i.e., the dominant global response alternative, see Table I; and the local default group, see Table II). At the same time it consistently reduced latency for the nondominant response. Future research should investigate whether the activation of the system (extension memory) that provides response alternatives is associated with an inhibition of the dominant response alternative.

\section{CONCLUSION}

To conclude, the present findings extend previous research on positive affect and flexibility obtained in decision making (Estrada et al., 1997), problem solving (Isen, 2004), and creativity (Isen, 1999b) to the domain of information processing: Positive affect can facilitate cognitive flexibility and enable individuals to overcome the precedence of global over local information processing when the task requires local processing.

\section{ACKNOWLEDGEMENT}

This work was supported by a grant from the German Science Foundation (DFG: Ku 377/22-1) to Julius Kuhl. We thank Miguel Kazén for helpful comments on a previous version of this article.

\section{REFERENCES}

Basso, M. R., Schefft, B. K., Ris, M. D., \& Dember, W. M. (1996). Mood and global-local visual processing. Journal of the International Neuropsychological Society, 2, 249-255.

Baumann, N., \& Kuhl, J. (2002). Intuition, affect, and personality: Unconscious coherence judgments and self-regulation of negative affect. Journal of Personality and Social Psychology, 83, 12131223. 
Beeman, M., Friedman, R. B., Grafman, J., Perez, E., Diamond, S., \& Lindsay, M. B. (1994). Summation priming and coarse semantic coding in the right hemisphere. Journal of Cognitive Neuroscience, 6, 26-45.

Bodenhausen, G. V., Kramer, G. P., \& Susser, K. (1994). Happiness and stereotypic thinking in social judgment. Journal of Personality and Social Psychology, 66, 621-632.

Bolte, A., Goschke, T., \& Kuhl, J. (2003). Emotion and Intuition: Effects of positive and negative mood on implicit judgments of semantic coherence. Psychological Science, 14, 416421.

Clore, G. L., Gasper, K., \& Garvin, E. (2001). Affect-as-information. In J. P. Forgas (Ed.), Handbook of affect and social cognition (pp. 121-144). Mahwah, NJ: Erlbaum.

Derryberry, D., \& Reed, M. A. (1998). Anxiety and attentional focusing: Trait, state and hemispheric influences. Personality and Individual Differences, 25, 745-761.

Dreisbach, G., \& Goschke, T. (2004). How positive affect modulates cognitive control: Reduced perseveration at the cost of increased distractibility. Journal of Experimental Psychology: Learning, Memory, and Cognition, 30, 343-353.

Epstein, S., Pacini, R., Denes-Raj, V., \& Heier, H. (1996). Individual differences in intuitive-experiential and analytical-rational thinking styles. Journal of Personality and Social Psychology, 71, 390405.

Estrada, C. A., Isen, A. M., \& Young, M. J. (1997). Positive affect facilitates integration of information and decreases anchoring in reasoning among physicians. Organizational and Human Decision Processes, 72, 117-135.

Gasper, K. (2004). Do you see what I see? Affect and visual information processing. Cognition and Emotion, 18, 405-421.

Gasper, K., \& Clore, G. L. (2002). Attending to the big picture: Mood and global versus local processing of visual information. Psychological Science, 13, 34-40.

Isen, A. M. (1999a). Positive affect. In T. Dalgleish \& M. J. Power (Eds.), Handbook of cognition and emotion (pp. 521-539). New York: Wiley \& Sons.

Isen, A. M. (1999b). Positive affect and creativity. In S. Russ (Ed.), Affect, creative experience, and psychological adjustment (pp. 3-17). Philadelphia: Bruner/Mazel.

Isen, A. M. (2001). An influence of positive affect on decision making in complex situations: Theoretical issues with practical implications. Journal of Consumer Psychology, 11, 75-85.

Isen, A. M. (2004). Some perspectives on positive feelings and emotions: Positive affect facilitates thinking and problem solving. In A. S. R. Manstead, N. Frijda, \& A. Fischer (Eds.), Feelings and emotions: The Amsterdam symposium (pp. 263-281). Cambridge, NY: Cambridge University Press.

Isen, A. M., Johnson, M. M. S., Mertz, E., \& Robinson, G. F. (1985). The influence of positive affect on the unusualness of word associations. Journal of Personality and Social Psychology, 48, 1413-1426.

Kahn, B. E., \& Isen, A. M. (1993). The influence of positive affect on variety seeking among safe, enjoyable products. Journal of Consumer Research, 20, 257-270.

Kazén, M., \& Kuhl, J. (2005). Needs and the modulation of cognitive systems: Volitional facilitation and inhibition as a function of affective contents of need-related stimuli. Journal of Personality and Social Psychology, 89(3).

Kazén, M., \& Kuhl, J. (2005). Affect regulation in a missing figure task: The role of strategic and personality factors. Manuscript submitted for publication. University of Osnabrück, Germany.

Kimchi, R. (1992). Primacy of wholistic processing and global/local paradigm: A critical review. Psychological Bulletin, 112, 24-38.

Kimchi, R., \& Palmer, S. E. (1982). Form and texture in hierarchically constructed patterns. Journal of Experimental Psychology: Human Perception and Performance, 8, 521-535.

Koole, S. L., \& Kuhl, J. (2003). In search of the real self: A functional perspective on optimal self esteem and authenticity. Psychological Inquiry, 14, 43-49.

Koole, S., \& Jostmann, N. (2004). Getting a grip on your feelings: Effects of action orientation and external demands on intuitive affect regulation. Journal of Personality and Social Psychology, 87, 974-990.

Kuhl, J. (2000). A functional-design approach to motivation and self-regulation: The dynamics of personality systems interactions. In M. Boekaerts, P. R. Pintrich \& M. Zeidner (Eds.), 
Self-regulation: Directions and challenges for future research (pp. 111-169). New York: Academic Press.

Kuhl, J. (2001). Motivation und Persönlichkeit [Motivation and personality]. Göttingen, Germany: Hogrefe.

Kuhl, J., \& Kazén, M. (1999). Volitional facilitation of difficult intentions: Joint activation of intention memory and positive affect removes stroop interference. Journal of Experimental Psychology: General, 128, 382-399.

Navon, D. (1977). Forest before trees: The precedence of global features in visual processing. Cognitive Psychology, 9, 353-383.

Navon, D. (1983). How many trees does it take to make a forest? Perception, 12, 239-254.

Rotenberg, V. S. (1993). Richness against freedom: Two hemisphere functions and the problem of creativity. European Journal for High Ability, 4, 11-19.

Schwarz, N. (2002). Feelings as information: Mood influence judgment and processing strategies. In T. Gilovich, D. Griffin, \& D. Kahnemann (Eds.), Heuristic and biases: The psychology of intuitive judgment (pp. 534-547). Cambridge, NY: Cambridge University Press.

Wheeler, M. A., Stuss, D. T., \& Tulving, E. (1997). Toward a theory of episodic memory: The frontal lobes and autonoetic consciousness. Psychological Bulletin, 121, 331-354. 